\title{
Teaching medical students on Surgical placement during the COVID-19 pandemic, lessons learned so far
}

\author{
Evans, W. and Noone, T.M.
}

\begin{abstract}
Background: The COVID-19 pandemic has caused significant disruption to the teaching and progression of medical students. With no imminent end to the pandemic, it remains vital that medical students remain integrated into clinical environments to benefit from the teaching opportunities in the hospital setting.
\end{abstract}

Aim: To offer advice on the teaching of medical students undertaking hospital placements within General Surgery during the COVID-19 pandemic.

Method: We reflected on our experience as clinical medical educators working through the COVID-19 pandemic, supported by feedback from medical students and relevant contemporary educational guidelines and literature.

Results: A review on how to optimise teaching medical students during the COVID-19 pandemic, balancing the need for safety and limiting viral transmission, while maximising clinical experience and learning.

Conclusion: The COVID-19 pandemic has forced widespread changes to the way in which medical students receive teaching. We suggest ways to maximise learning opportunities, on hospital placements, during the pandemic. Maintaining quality medical education during the pandemic is key to producing the best doctors of tomorrow.

Keywords: Covid-19, Medical education, Novel teaching, Practical and Clinical tutoring, Undergraduate

\section{Introduction}

The COVID-19 pandemic has severely disrupted medical student education worldwide. In an effort to reduce viral transmission, clinical teaching has faced cancellation or conversion to online internet-based platforms to replace traditional face-to-face methods. As we enter the second year of the COVID-19 pandemic, it is evident that medical education will continue to be affected for a significant time to come

General Surgery, Northwest Post Graduate Medical Deanery, Manchester, UK

Corresponding author: William Evans mbchfwe2@doctors.org.uk

DOI: http://doi.org/10.4038/seajme.v15i2.356
(Co \& Chu, 2020; Al-Taweel et al, 2020; Hofmann et al, 2020), particularly with subsequent COVID-19 outbreaks and the emergence of new viral variants. This is confounded by further lockdowns and varying accessibility to vaccines. Despite these challenges, the importance of maintaining clinical teaching programmes has been emphasised by global health bodies (UNESCO, 2020) and the UK Medical Schools Council, who recognise "The next challenge is to ensure that a full cohort of students receive the education that they need to graduate in August 2021 and that students in the earlier years of their course also receive appropriate training to allow them to progress" (MSC 2021). 
Transition to online platforms has facilitated aspects of medical education during the initial phase of the COVID-19 pandemic, however it has become apparent that preparedness for medical school examinations has been detrimentally affected (Choi et al, 2020). This shortfall is potentially related to suspension of medical students from hospitals, with reduction in exposure to the learning opportunities provided by the clinical environment. Surgery is a practical discipline, requiring students to acquire clinical skills that are difficult to emulate in a virtual learning environment. The full impact of the changes to medical education, during the COVID-19 pandemic, with regards to the performance of newly qualified doctors in the UK Foundation Training programme, remains unclear.

Teaching in the clinical environment offers a number of advantages over online platforms. It aids the development of medical students helping to bridge theory to clinical practice and to gain competency in core clinical skills. Clinical teaching has also been shown to improve examination performance, correlate with empathy towards patients and future career decision-making ability (Dornan et al, 2006). One example, the longstanding concept of 'bedside teaching,' uses hospital inpatients as case studies for the education of medical students. This promotes learning through association, application of knowledge and development of clinical history and physical examination skills. Currently used online platforms cannot replicate this comprehensively. The benefits of bedside teaching result in it being a preferred method for educators, students and patients alike (Narayanan, 2020).

In the United Kingdom, standard medical student education in the clinical environment, is beginning to return. In-person 'Objective Structured Clinical Examinations' (OSCEs) have restarted, albeit with actors or other medical students as simulated patients, rather than actual patients. Students have also largely returned to the clinical ward environment since the commencement of the 2020-21 academic year. However, this has been associated with significant logistical difficulties due to stringent restrictions on numbers of medical students permitted in one clinical area at a given time. In addition, the substantial resources and hospital capacity devoted to patients with SARS-CoV-2 infection, has diminished other services, such as elective surgery and face-to-face outpatient clinics. Overall, this has reduced the diversity of learning opportunities available to medical students.

Within our practice of General Surgery, we have seen widespread cancellation of outpatient clinics in favour of telephone consultations, rationalisation of operating lists, endoscopy and screening services, plus necessary but prohibitive infection control restrictions imposed on daily ward rounds. Educating medical students in these changing circumstances has been challenging; we share our experience of how to maximise learning opportunities for medical students on clinical placements during the COVID-19 pandemic.

\section{Ensure physical and mental wellbeing}

The first action in returning students to the clinical environment is to ensure the safety of both patients and students, while adhering to infection control measures. The Medical Schools Council (MSC) has issued guidance on this and continues to update it as the COVID19 pandemic develops. At an individual level, all students should complete a COVID-specific risk assessment prior to commencing a hospital placement. The Medical School and/or the Hospital's Occupational Health Department should be informed of any concerns or changes to level of risk. Medical Schools should assess risk for specific clinical placements and make safety modifications accordingly. Students should be assigned to placements with consideration of these risks; with high-risk students allocated to areas of lowest infection prevalence/risk (MSC, 2021).

The MSC recommends that students should be considered high priority for vaccination and be included in local policies with regards to personal protective equipment (PPE), such as access to launderable 'scrubs' type clothing. They ask that Medical Schools are accommodating in deferring the studies of 
students considered at high risk from a physical or mental health point of view (MSC, 2021).

Stress in medical students is shown to be worse than the general public (Bergmann et al., 2019). This is expected to be exacerbated during the COVID-19, due to the effects of the pandemic itself, as well as disruptions to medical education and examinations (O'Byrne et al., 2021). Concerns raised by local medical students included "Will I get the training I require?", "How will I learn the clinical skills I need?", "Will I become unwell if I contract COVID on placements?"

It is recognised that stress affects academic performance, physical health and mental wellbeing. It is therefore more important than ever to recognise, prevent and manage stress. One strategy is to use a questionnaire to screen for stress during educational supervisor meetings. Advice can then be given on the strategies commonly used to manage stress during the pandemic, including video chats, exercise and fitness, hobbies and routine and goal setting (Lyons et al., 2020). More structured intervention can include mindfulness practice, with self-directed techniques suitable to the pandemic, and shown to reduce stress and anxiety in medical students (Warnecke et al., 2011).

Identify learning objectives and adjust competency expectations accordingly Medical students should meet with their clinical educational supervisors (ES) at the start of the placement to agree learning objectives. Induction meetings should be structured and agenda driven. Supervisors can achieve this with a "SMART-GEM" model (Bowman et al, 2015). This guides both the trainer and student towards setting Specific objective, which can be Measured, are Attainable, Relevant to their learning and achievable during a realistic Time frame. This Goal Evaluation Method encourages focus, countering the challenging teaching and learning circumstances that the pandemic has created.

Interim reviews should be held early, with additional meetings encouraged and facilitated as needed. Early review and feedback from medical students is essential, because students are now more likely to experience novel frustrations to their learning objectives. This will allow for different learning objectives to be set if COVID related learning obstacles are insurmountable. "Regular meetings help development of a strong relationship between the trainee and ES, and can help the ES to build a picture of the trainee's development and progress, as well as giving the trainee support" (Wainman, 2018)

Medical student teaching at all levels has had to be adjusted since the start of the COVID-19 pandemic. The didactic approach of online platforms is adequate for teaching theory, clinical history taking and discussing management plans. However, a significant limiting factor of this style of teaching is with clinical elements of medical education, such as physical examination and practical skills. Before the pandemic, medical students would be expected to maintain a logbook of their experience performing physical examinations and practical skills such as phlebotomy, cannulation and insertion of a urinary catheter. With students temporarily suspended from clinical areas, this has not always been possible during the pandemic. Should medical students encounter difficulty getting experience in doing a particular skill, logbook number requirements should be adjusted and alternative methods of assessing competency should be introduced (Chung et al, 2020).

\section{Utilise and focus on functioning services}

Although COVID-19 has had an impact across all medical specialities, some have been more affected than others. Initially, there was a disproportionately high impact on the specialities associated with 'aerosol generating procedures', such as Anaesthetics, Surgical specialties, Respiratory Medicine and Endoscopy services. For example, the National Endoscopy Database recorded an activity level of just $5 \%$ of normal during the pandemic (Rees et al, 2020). High risk procedures and services were either stopped or reduced to minimise risks of exposure to and transmission of COVID-19. Consequently, educational 
opportunities in these fields were vastly reduced.

Although restrictions on these specialities have eased or been overcome with PPE, on-going safety precautions have reduced patient workload or precluded the attendance of medical students' altogether (Phillips et al, 2020). Within General Surgery, there was initially a reduction in elective and minimally invasive surgery (Garg, 2021), however emergency services have largely continued as normal throughout the pandemic. This has proved a valuable source of learning for medical students, engaging them in all aspects of the emergency patient journey, from initial presentation through to definitive management. Different medical students were attached to the various elements of the inpatient experience, to maximise their clinical exposure to services still functioning. Additionally, we were able to introduce students to more severe or advanced forms of acute surgical pathologies presenting as a result of delays caused by the pandemic (Guadalajara et al, 2020).

Medical students should seek to gain experience and learning opportunities from all allied healthcare professional colleagues; for example, a substantial amount of medical student education is successfully delivered by nursing colleagues (Gilmour et al, 2014). Suitable members of the multi-disciplinary team include advanced nurse practitioners, specialist nurses, physician associates, physiotherapists and pharmacists.

While a medical student is attached to a General Surgery hospital placement there are clear benefits in seeing how interlinked services and MDT members work. This promotes a broader understanding of the specialty and the individual roles of team members. By involving MDT members in teaching medical students, more students can be allocated to different clinical areas within the hospital, therefore allowing a wider distribution of students. This also theoretically helps to reduce transmission of COVID-19, by minimising congregation and crowding in clinical areas. Student contact time can be staggered throughout the day. Conventionally, most medical staff are present in the hospital throughout daytime working hours, meaning that most learning opportunities available to medical students are during the day. Throughout the pandemic, national guidance has prompted a review of the hospital workforce, with a move towards remote working where appropriate (National Institute of Health and Care Excellence (NICE), 2020). After the initial suspension of medical students from hospitals, there has been a gradual reintroduction with the recognition that their training must continue (MSC, 2021). In order to maintain safety, the numbers of students permitted at any given time has been dramatically reduced. Therefore, to share teaching opportunities amongst all students, strict timetables have been implemented, spreading students throughout the day. For example, one student attending the morning session of an operating list and then swapping with another student to attend the afternoon session. This allows two students to benefit from the clinical experience, learning how to scrub-up and assisting in theatre, through the division of a single operating list.

Although not routinely offered, there are benefits to medical students attending the hospital outside of regular daytime hours, supported by the hospital on-call team at night (Goren, 2015). Intensity of work during out-ofhours shifts can be variable, which creates opportunities with either more time for directed teaching, or learning from clinical cases during busier periods. Following guidance to optimise overnight teaching is advised (Richards et al., 2013).

Aim to maximise the effectiveness of clinical contact time

While on clinical placement, all time can be utilised for learning, not just when being actively taught. Shadowing a junior doctor performing their role allows medical students to familiarise themselves with the job role they are training for and has been to shown to improve patient safety (Pathiraja \& Outram, 2011).

At the start of the COVID-19 pandemic, when final year medical students were due to undertake medical elective placements abroad, 
the restrictions on international travel curtailed one opportunity while leading to the creation of another - the Foundation interim Year 1 (FiY1). This voluntary, paid role allowed final year medical students the opportunity to work oneto-one with a Foundation Year 1 doctor, minimising their missed educational opportunities and downtime. Although challenging to implement, this offered additional clinical experience, opportunity to become accustomed to new responsibilities and enhanced their portfolios (Youssef et al., 2020).

WBAs are an important aspect of clinical teaching and have been described as "a powerful means of changing a student's behaviour" (Norcini \& Burch, 2007). Many students are often assigned a 'clinical partner' during their attachment for the purposes of peer learning. Each student can be encouraged to focus on one element of the patient interaction, which they can then be assessed on independently. For instance, a single patient consultation can be broken down into clinical history, physical examination, differential diagnosis and management options. This can rationalise the number of patients a given student comes into contact with, yet still form a substantial basis for a teaching and learning, through several WBAs.

In order for WBAs to be effective, constructive feedback is crucial (Norcini \& Burch, 2007). Often, this is the most variable part of WBAs (Boulet et al, 2002) and is difficult to standardise. Therefore, it is important that teachers have adequate training with regard to constructive feedback techniques. Additionally, student self-reflective feedback is another important element of WBAs (Hornbloe et al, 2004). This process of reflection, consolidation and building upon learning points generated by the WBA, maximises the teaching value.

\section{Encourage the development of non-clinical skills}

With restrictions placed on medical students' attendance in hospital, more time is available to focus on non-clinical skills that are required by modern doctors. One such skillset, mandatory to continuing professional development and imperative to career progression, is involvement in audit and medical research. Departmental journal clubs are an opportune environment to promote the practice of evidence-based medicine, and enable medical students' development of both presentation and critical appraisal skills, as recommended by the General Medical Council (GMC) (GMC, 2009). During the pandemic, journal clubs can move to an online platform in order to maintain infection control measures.

With more time available for non-clinical activities, students should be encouraged to get involved in research and audit, which they have historically struggled to engage with. One example is STARsurg, a national student-led research collaborative. This engagement with research has been demonstrated to improve their confidence and acquisition of non-clinical skills (Chapman et al., 2015). Encouraging medical student involvement in audit and research will prove invaluable during their careers, aiding their path to future audits, publications or even post-graduate degrees such as PhDs.

\section{Share knowledge, seek feedback}

With varying seniority of medical students attached to a clinical placement, it is possible to combine the different student levels together for clinical teaching sessions. For example, with bedside teaching, include all levels within a small group, enabling the students to learn different elements or objectives from one clinical encounter. Students should be encouraged to share knowledge and give feedback to each other.

During the COVID-19 pandemic, we have found peer assisted learning to be invaluable. The benefits relate to its inclusivity, flexibility, ability to be structured or unstructured, and suitability to different formats. It is an excellent method of sharing knowledge through structured formats such as bedside teaching, and unstructured formats such as students debriefing cases during break time and observation of peer practice (Tai et al., 2014) 


\section{Conclusion}

The COVID-19 pandemic forced widespread changes to the way in which medical students receive teaching and it continues to be a dynamic and evolving situation. We have summarised important points from our pandemic experiences, to provide clinical medical educators with techniques to maximise learning opportunities for medical students on hospital placement. Maintaining quality medical education during pandemics is vital to produce the high standard of doctors we require, because "medical students are tomorrow's doctors" (GMC, 2018).

Declaration of interest: The authors report no declaration of interest. The authors alone are responsible for the content and writing of the article.

\section{References}

Al-Taweel, D., Al-Haqan, A., Bajis, D., Al-Bader, J., Al-Taweel, A.M., Al-Awadhi, A. \& Al-Awadhi, F. 2020, "Multidisciplinary academic perspectives during the COVID-19 pandemic", The International journal of health planning and management, vol. 35, no. 6, pp. 1295-1301.

Bergmann, C., Muth, T. \& Loerbroks, A. 2019, Medical students' perceptions of stress due to academic studies and its interrelationships with other domains of life: a qualitatitve study. Medical Education Online, vol 24 (1), 1603526

Boulet JR, Mckinley DW, Norcini JJ, Whelan GP. Assessing the comparability of standardized patient and physician evaluations of clinical skills. Ad Health Sci Educ 2002; 7: 85-97.

Bowman, J., Mogensen, L., Marsland, E. \& Lannin, N. 2015, "The development, content validity and inter-rater reliability of the SMART-Goal Evaluation Method: A standardised method for evaluating clinical goals", Australian occupational therapy journal, vol. 62 , no. 6 , pp. 420-427.

Chapman SJ, Glasbey JC, Khatri C, Kelly M, Nepogodiev D, Bhangu A, Fitzgerald JE. 2015, "Promoting research and audit at medical school: evaluating the educational impact of participation in a student-led national collaborative study". BMC Med Educ. 13; 15:47.

Choi, B., Jegatheeswaran, L., Minocha, A., Alhilani, M., Nakhoul, M. \& Mutengesa, E. 2020 "The impact of the COVID-19 pandemic on final year medical students in the United Kingdom: a national survey", BMC Medical Education 20, 206
Chung, P., Tsang, A., Co, M. \& Chu, K. 2020, "Assessing clinical competency without patients during COVID-19 pandemic" Surgical practice, vol 24, no. 4, pp. 156-156.

Co, M. \& Chu, K. 2020, "Distant surgical teaching during COVID-19 - A pilot study on final year medical students", Surgical practice, vol. 24, no. 3, pp. 105-109.

Dornan, T., Littlewood, S., Margolis, S.A., Scherpbier, A., Spencer, J. \& Ypinazar, V. 2006, "How can experience in clinical and community settings contribute to early medical education? A BEME systematic review", Medical teacher, vol. 28, no. 1, pp. 3-18.

Dost, S., Hossain, A., Shehab, M., et al. 2020. "Perceptions of medical students towards online teaching during the COVID-19 pandemic: a national cross-sectional survey of 2721 UK medical students". BMJ Open, 10, e042378.

Garg, H. 2021, "COVID-19 and jeopardy of a trainee in the era of minimally invasive surgery", Journal of minimal access surgery, vol. 17, no. 1, pp. 135136.

Gilmour, J., Huntington, A., Bogossian, F., Leadbitter, B. \& Turner, C. 2014, "Medical education and informal teaching by nurses and midwives", International journal of medical education, vol. 5, pp. 173-177.

General Medical Council, 2009, Tomorrow's Doctors. General Medical Council, London

General Medical Council, 2018, Outcomes for Graduates. General Medical Council, London. Available at: https://www.gmcuk.org/education/standards-guidance-andcurricula/standards-and-outcomes/outcomesfor-graduates (Accessed on: 25th May 2021)

Goren, E., Leizman, D., La Rochelle, J. \& Kogan, J. 2015, Overnight Hospital Experiences for Medical Students: Results of the 2014 Clerkship Directors in Internal Medicine National Survey, Journal of General Internal Medicine, vol 30, pp $1245-1250$

Guadalajara, H., Muñoz de Nova, J., Fernandez Gonzalez, S., Yiasemidou, M., Recarte Rico, M., Juez, L., García Septiem, J., Galindo Jara, P., García Virosta, M., Lobo Martínez, E., MartínPérez, E., García-Olmo, D. \& PIACO Collaboration Group 2020, "Patterns of acute surgical inflammatory processes presentation of in the COVID-19 outbreak (PIACO Study): Surgery may be the best treatment option", British Journal of Surgery, 107, 11, pp.e494e495.

Hofmann, H., Harding, C., Youm, J. \& Wiechmann, W. 2020, "Virtual bedside teaching rounds with patients with COVID-19", Medical education, vol. 54, no. 10, pp. 959-960. 
Holmboe ES, Yepes M, Williams F, Huot SJ. Feedback and the mini-clinical evaluation exercise. J Gen Inter Med 2004a; 19: 558-561

Lalvarmawi, F., Banik, U \& Devi, M. 2015, Feedback of medical students on teaching and evaluation methodology in Physiology. National Journal of Physiology, Pharmacy and Pharmacology, 5, 1, pp36-38.

Lyons Z, Wilcox H, Leung L, Dearsley O. 2020. "COVID-19 and the mental well-being of Australian medical students: impact, concerns and coping strategies used". Australas Psychiatry. 28(6):649-652.

Mandouit, L. 2018. Using student feedback to improve teaching, Educational Action Research, 26, 5, pp755-769

Medical Schools Council (MSC), 29/01/2021. Updated statement on clinical placements.

Medical Schools Council (MSC), 29/01/2021. Statement on 'Returning to clinical placements risk assessments'.

Narayanan, V. and Nair, B. R. (2020) 'The value of bedside teaching in undergraduate medical education: a literature review'

National Institute for Health and Care Excellence (NICE), NHS England, 2020, Clinical guide for the management of remote consultations and working in secondary care during the coronavirus pandemic. Available at: https://www.nice.org.uk/Media/Default/About/CO VID-19/Specialty-guides/specialty-Guide-VirtualWorking-and-Coronavirus.pdf (Accessed: 24th May 2021)

Norcini, J. \& Burch, V. 2007, "Workplace-based assessment as an educational tool: AMEE Guide No. 31", Medical teacher, vol. 29, no. 9-10, pp. 855-871.

O’Byrne, L., Gavin, B., Adamis, D., et al. 2021. "Levels of stress in medical students due to COVID-19". Journal of Medical Ethics, vol 47 pp383-388.

Pathiraja, F., Outram, C. 2011, Making the case for shadowing, BMJ, 342:d142.
Phillips, G., Jones, M. \& Dagg, K. 2020, "Restarting training and examinations in the era of COVID19: a perspective from the Federation of Royal Colleges of Physicians UK", Clinical medicine (London, England), vol. 20, no. 6, pp. clinmed.2020-0481-e252.

Rees, C.J., East, J.E., Oppong, K., Veitch, A., McAlindon, M., Anderson, J., Hayee, B., Edwards, C., McKinlay, A. \& Penman, I. 2020, "Restarting gastrointestinal endoscopy in the deceleration and early recovery phases of COVID-19 pandemic: Guidance from the British Society of Gastroenterology", Clinical medicine (London, England), vol. 20, no. 4, pp. 352-358.

Richards, J., Wilcox, S., Roberts, D. \& Schwartzstein, R. 2013, Twelve tips for overnight teaching, Medical Teacher, vol 36, no. 3, pp 196200

Stout, R., Roberts, S., Maxwell-Scott, H., et al. 2021., Necessity is the mother of invention: how the COVID-19 pandemic could change medical student placements for the better. Postgraduate Medical Journal, 0, pp1-6.

Tai, J., Haines, T., Canny, B. \& Molloy, E. 2014, A study of medical students peer learning on clinical placements: What have they taught themselves to do, Journal of Peer Learning, vol 7, pp57-80

UNESCO, 2020. COVID-19 educational disruption and response.

Wainman, P. 2018, "Crammer's Corner: Preparing for educational supervisor meetings", InnovAiT, vol. 11 , no. 2, pp. 115-116

Warnecke, E., Quinn, S., Ogden, K. et al. 2011, A randomised controlled trial of the effects of mindfulness practice on medical student stress levels. Medical Education, vol 45 pp 381-388.

Youssef, S., Zaidi, S., Shrestha, S,. Varghese, C. \& Rajagopalan, S. 2020, First impressions of the foundation interim year 1 postings: positives, pitfalls, and perils, Medical Education Online, vol 25 , no. 1,1785116 . 